Supporting Information

\title{
Cyanobacteria affect fitness and genetic structure of experimental Daphnia populations
}

Bogdan Drugă ${ }^{* 1,2}$, Patrick Turko ${ }^{1,3}$, Piet Spaak ${ }^{1,3}$, Francesco Pomati ${ }^{1,3}$

${ }^{1}$ Eawag, Swiss Federal Institute of Aquatic Science and Technology, Department of Aquatic Ecology, 133 Überlandstrasse, 8600 Dübendorf, Switzerland

${ }^{2}$ Institute of Biological Research, branch of the National Institute of Research and Development for Biological Sciences, 48 Republicii, 400015 Cluj-Napoca, Romania

${ }^{3}$ Institute of Integrative Biology, ETH Zurich, Universitätstrasse 16, 8092 Zürich, Switzerland

\section{Section 1}

\section{Population growth rate}

Each bootstrap replicate began by randomly choosing (with replacement) individuals of clone-treatment population under question to create a pseudo-population of the same size as the experimental population. The birth-death data of these individuals was combined to construct a Leslie matrix, and the largest real component of the matrix's dominant eigenvector, which corresponds to $\lambda$, was extracted and saved. This process was repeated 1000 times for each clone-treatment group. The resulting population growth rates were analyzed using a modified ANOVA, wherein the treatment and error sums of squares (SS) were calculated as usual, but the Mean Square values, the F-values and the p-values were calculated using residual degrees of freedom derived from the actual size of the experiment. This procedure yields the same results regardless of the number of bootstrap replicates.

\section{RNA concentration}

RNA concentration was measured with a Qubit 2.0 fluorometer (Life Technologies, Carlsbad, CA). To remove any trace of genomic DNA, samples were treated with 1 unit DNase (Ambion Turbo DN-ase, Austin, TX). Synthesis of first strand cDNA was performed using a First Strand cDNA Synthesis Kit (Thermo Scientific, Waltham, MA) using $1.5 \mu \mathrm{g}$ of purified RNA as template and random hexamer primers. 


\section{qPCR}

For the qPCR, SensiFast SYBR \& Fluorescein Kit (Bioline, London, UK) was used, with the reaction mix containing both forward and reverse primers at a concentration of 400 $\mathrm{nM}$ each, $10 \mu \mathrm{L}$ of SensiFAST SYBR \& Fluorescein Mix to a final concentration of $1 \mathrm{x}, 4 \mu \mathrm{L}$ of $5 x$ diluted cDNA and $\mathrm{H}_{2} \mathrm{O}$ added up to a final volume of $20 \mu \mathrm{L}$. The PCR profile contained: one cycle of $95{ }^{\circ} \mathrm{C}$ for $3 \mathrm{~min}$, followed by 45 cycles of $95{ }^{\circ} \mathrm{C}$ for $15 \mathrm{~s}, 55^{\circ} \mathrm{C}$ for 30 $\mathrm{s}$, and $72{ }^{\circ} \mathrm{C}$ for $30 \mathrm{~s}$. Negative controls (reactions without cDNA template) were included to detect the presence of any DNA contamination. All reactions were performed in triplicate.

\section{Microsatellite analysis}

The reactions for each $D$. galeata genotype was performed in a final volume of $11.5 \mu \mathrm{L}$, containing $0.3 \mu \mathrm{M}$ of respective forward and reverse primer (Supplementary table S2), using the Multiplex PCR Plus Kit (Qiagen, Venlo, Netherlands). The PCR parameters were: one cycle of $95^{\circ} \mathrm{C}$ for $15 \mathrm{~min}$ followed by 32 cycles of denaturation for $30 \mathrm{~s}$ at $94^{\circ} \mathrm{C}$, annealing for $90 \mathrm{~s}$ at $54^{\circ} \mathrm{C}$, and $60 \mathrm{~s}$ elongation at $72^{\circ} \mathrm{C}$. Final elongation was performed for $30 \mathrm{~min}$ at $60^{\circ} \mathrm{C}$. The analyses of the DNA fragment length were performed with a 3130 Genetic Analyzer (Applied Biosystems, Waltham, MA). 


\section{Section 2}

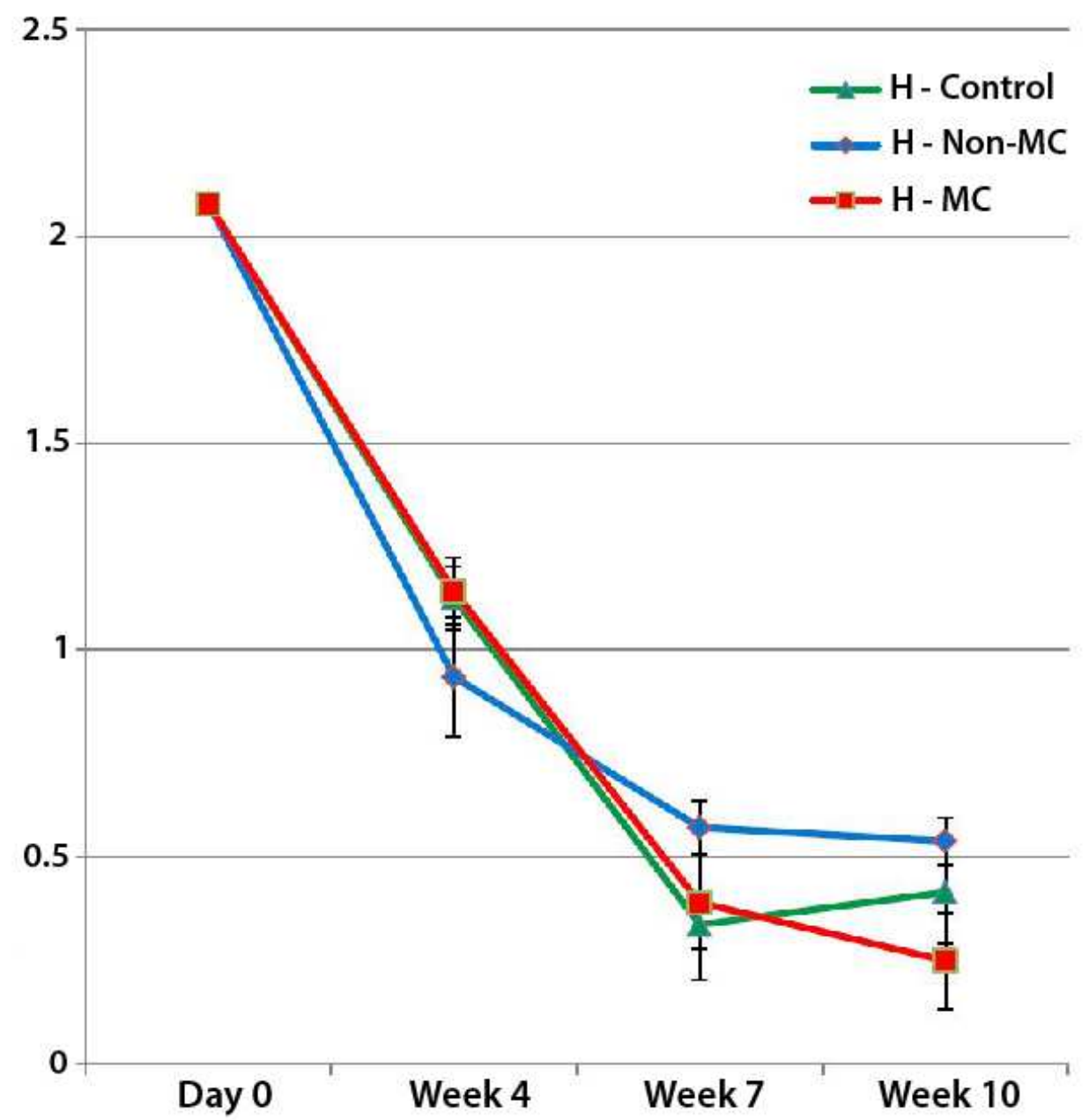

Supplementary Fig. S1 Population diversity of the D. galeata communities fed for ten weeks with $100 \% \mathrm{~S}$. obliquus (Control); 80\% S. obliquus $+20 \%$ M. aeruginosa PCC 7806_Non-MC and 80\% S. obliquus $+20 \%$ M. aeruginosa PCC 7806_MC. H = Shannon-Wiener index. 


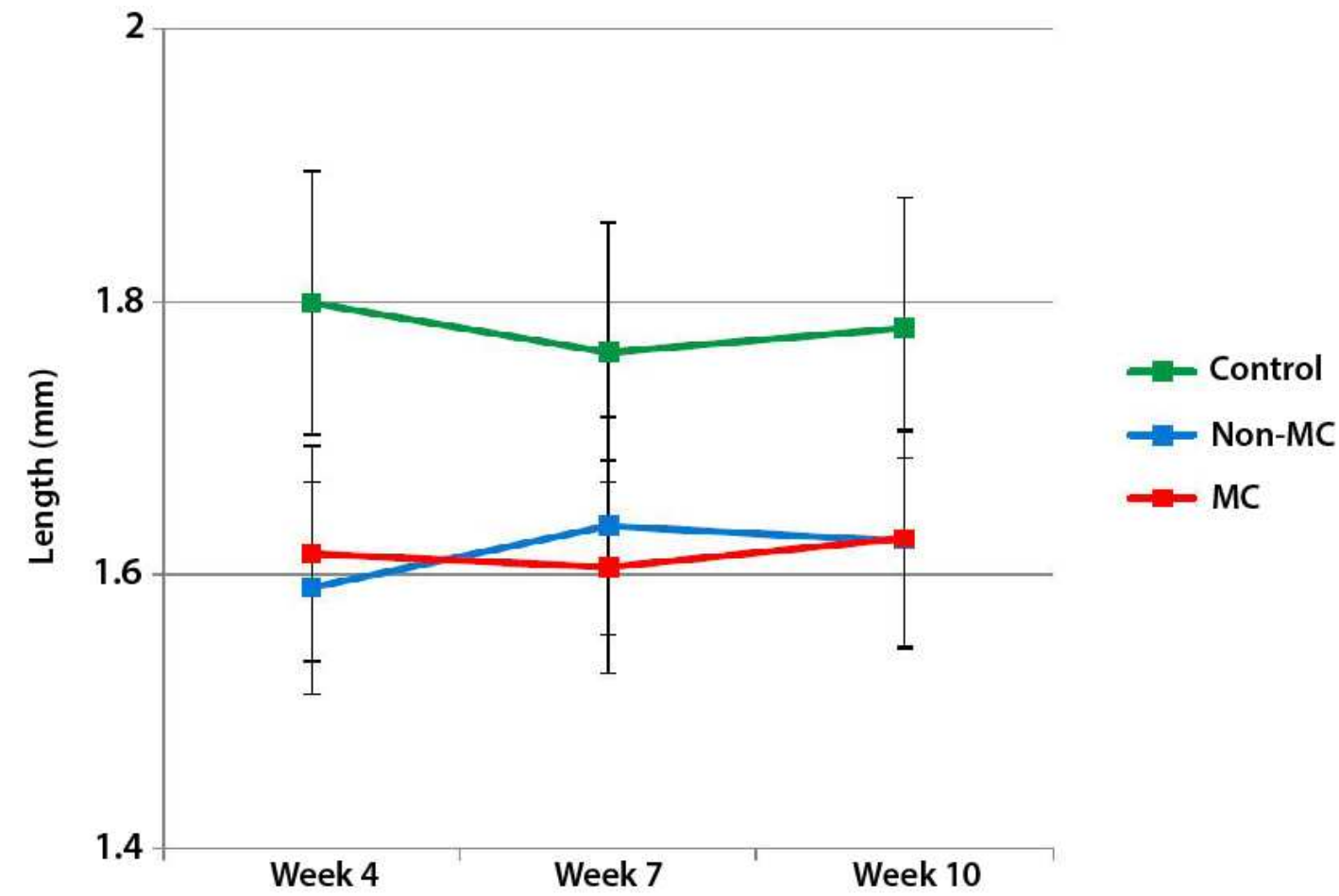

Supplementary Fig. S2 The body size of D. galeata individuals under control, non-MC, and MC-containing diets (see text) in the competition experiment. The bars represent the standard error calculated from the three replicates for each time point. 

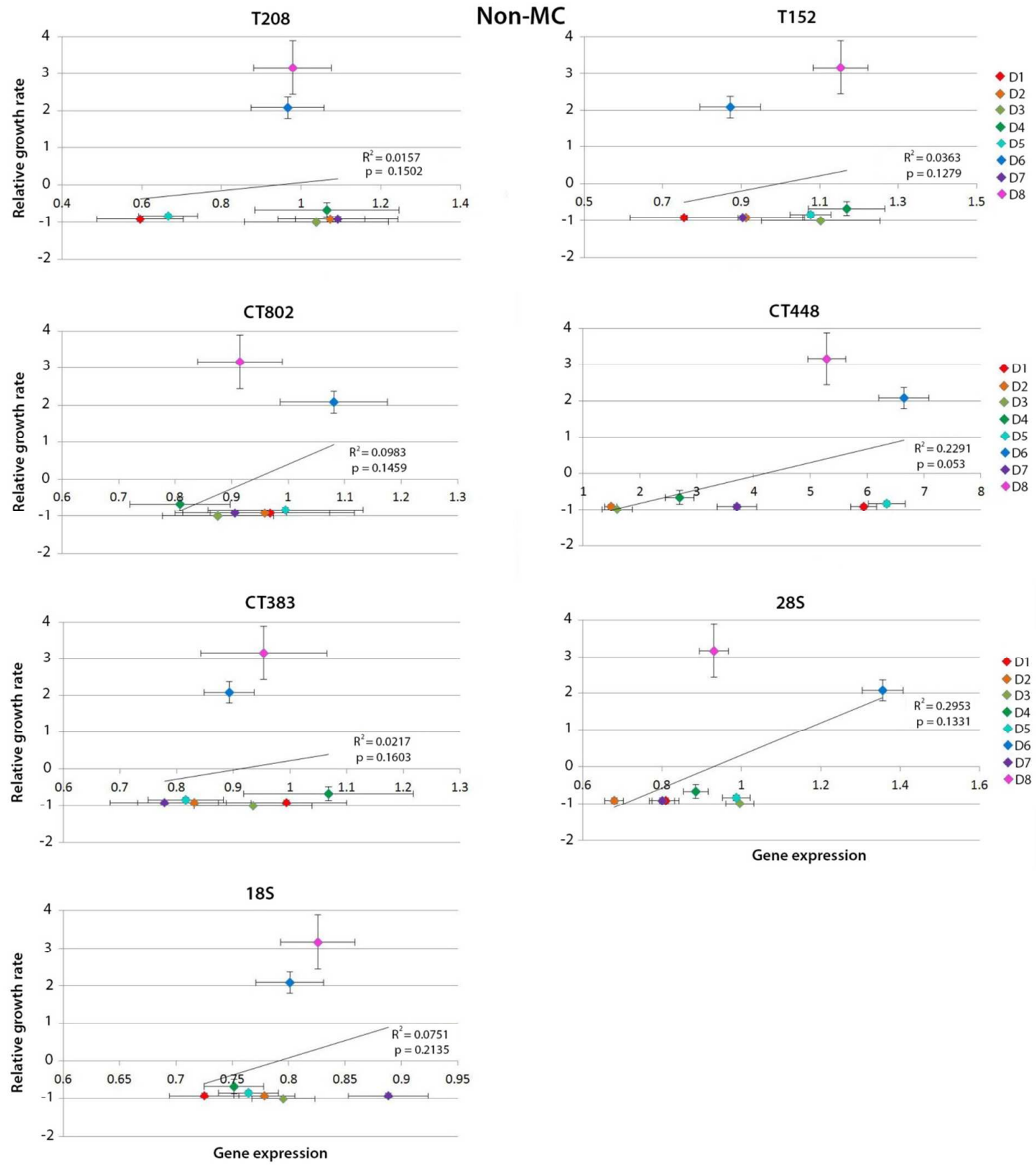

Supplementary Fig. S3 The expression of two trypsin (T208 and T152), three chymotrypsin (CT802, CT448 and CT383) and two ribosomal RNA (18S and 28S) genes plotted against the relative growth rates of the eight D. galeata clones in the competition experiment under non-MC conditions. The vertical and horizontal bars represent the standard error calculated for the relative growth rate and gene expression, using data from three replicates. 

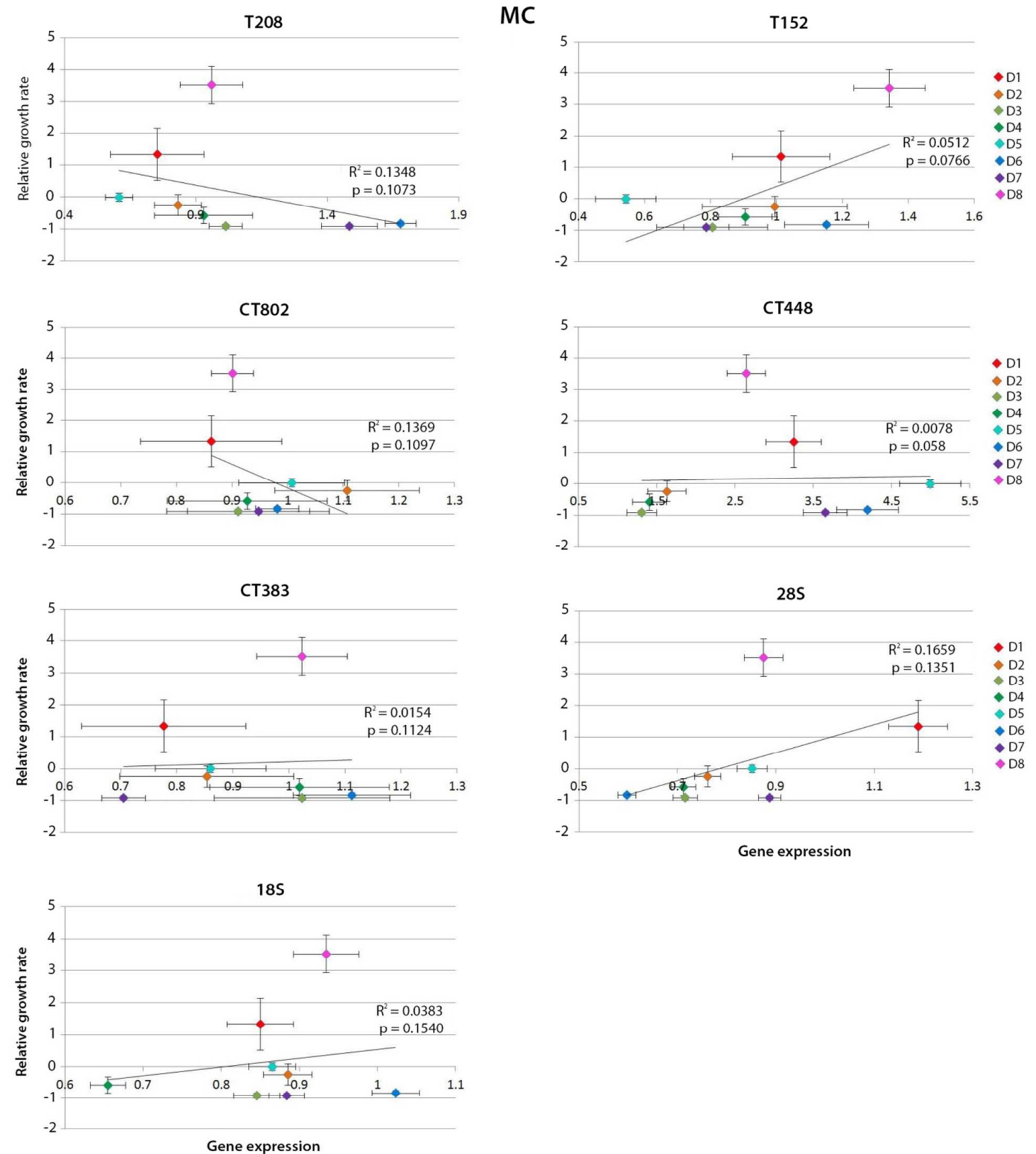

Supplementary Fig. S4 The expression of two trypsin, three chymotrypsin and two ribosomal RNA genes plotted against the relative growth rates of the eight $D$. galeata clones in the competition experiment with MCcontaining diet. 


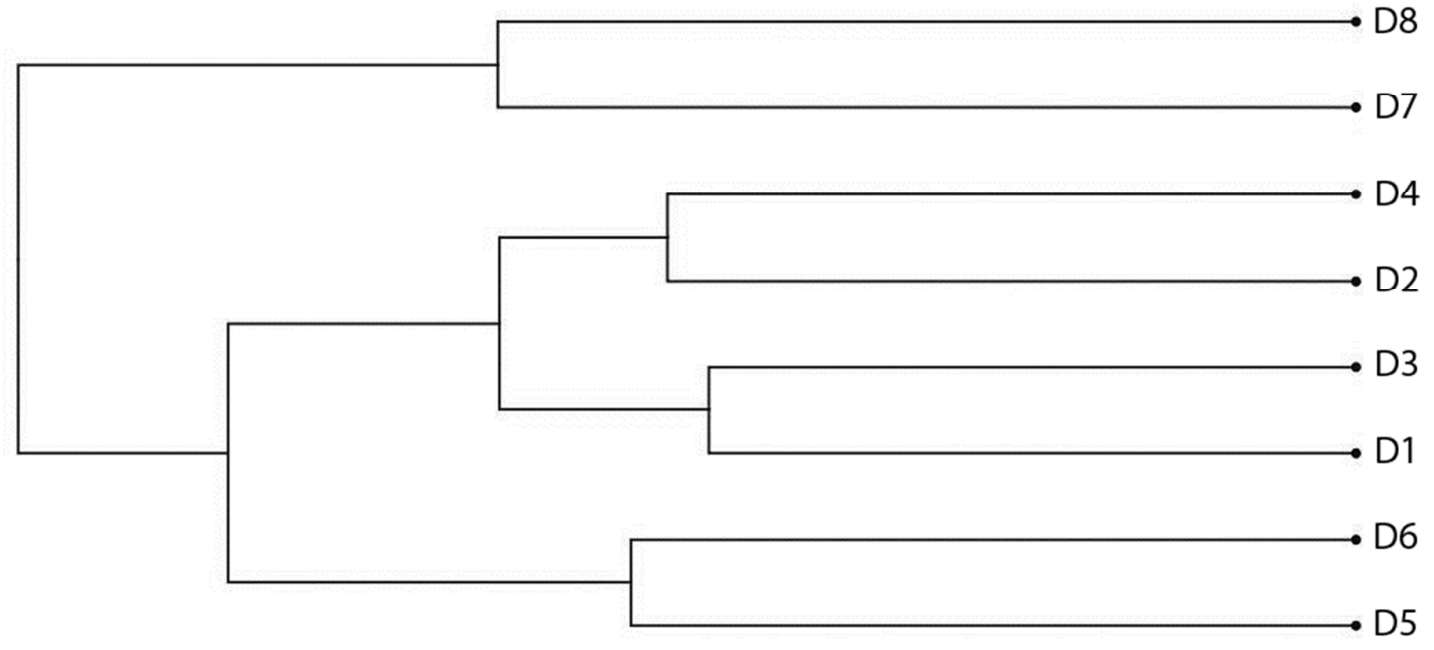

0.250

Relative distance

Supplementary Fig. S5 Dendrogram showing the relations between the eight $D$. galeata clones based on the microsatellites pattern. 


\section{Section 3}

Supplementary Tab S1 Sequences of the primers used for qPCR (Heckmann et al. 2006; Schwarzenberger et al. 2010). The gene names in bold $=$ the six candidate reference genes.

\begin{tabular}{|c|c|c|c|c|}
\hline Gene name & Symbol & Forward primer (5'-3') & Reverse primer (5'-3') & $\begin{array}{l}\text { Amplicon } \\
\text { size }\end{array}$ \\
\hline Actin & Act & CCACACTGTCCCCATTTATGAA & CGCGACCAGCCAAATCC & 71 \\
\hline Alpha-tubulin & Atb & TGGAGGTGGTGACGACT & CCAAGTCGACAAAGACAGCA & 89 \\
\hline Cyclophilin & Сур & GACTTTCCACCAGTGCCATT & AACTTTCCATCGCATCATCC & 78 \\
\hline $\begin{array}{l}\text { Glyceraldehyde-3- } \\
\text { phosphate DH }\end{array}$ & GapDH & GGCAAGCTAGTTGTCAATGG & TATTCAGCTCCAGCAGTTCC & 89 \\
\hline $\begin{array}{l}\text { Succinate } \\
\text { dehydrogenase }\end{array}$ & SDH & TGCCATTTAGTCGCACTCAG & GTGAGCTTGTCCTCCTTTGC & 89 \\
\hline $\begin{array}{l}\text { TATA-box binding } \\
\text { protein }\end{array}$ & TBP & GCAGGGAAGTTTAGTTTCTGGA & TGGTATGCACAGGAGCAAAG & 88 \\
\hline $\begin{array}{l}\text { Ubiquitin Conjugating } \\
\text { Enzyme }\end{array}$ & $\mathrm{UBC}$ & TCACCTGCACTCACCATTTC & AATCTCCGGAACCAAAGGAT & 90 \\
\hline $18 \mathrm{~S}$ ribosomal RNA & $18 \mathrm{~S}$ & CGCTCTGAATCAAGGGTGTT & TGTCCGACCGTGAAGAGAGT & 77 \\
\hline 28S ribosomal RNA & $28 \mathrm{~S}$ & GAGGCGCAATGAAAGTGAAG & TGTTCGAGACGGGATCA & 70 \\
\hline Trypsin 152 & $\mathrm{~T} 152$ & TGGATGCTCCATTGGACTTGA & CGGAAACGGTGACGATGATTC & 105 \\
\hline Trypsin 208 & $\mathrm{~T} 208$ & TGCGTTAGAGGAGTTGACGCT & TGAAGCTGACAACACCACGGT & 103 \\
\hline Trypsin 610 & $\mathrm{~T} 610$ & GGTGGCTCCATCTACAAGAG & ACCAGCAACGATGGACATG & 90 \\
\hline Chymotrypsin 383 & СТ383 & TTGGCACCTTCCACCGAAT & TCATCAGGACTGGAGAAACGC & 106 \\
\hline Chymotrypsin 448 & CT448 & TCATCAACGGAGCTGAGGCTA & AGAACCCACTGGTCGGAAATC & 109 \\
\hline Chymotrypsin 802 & СТ802 & TCAGATTGCCCAACCCCAT & TCCGCTGATGTGGAGCATATC & 104 \\
\hline
\end{tabular}


Supplementary Tab S2 The primers used for microsatellites analyses (Brede et al. 2006)

\begin{tabular}{lll}
\hline Microsatellite & Forward primer (5'-3') & Reverse primer (5'-3') \\
\hline SwiD1 & GCCGTGTTCGAAAGCTAGTC & AGCCGAACGAAAAACATGC \\
SwiD4 & GTCAAGTTGTTCTTGTTATTGTGC & TTTTGTAGGTCCGCGTAAATG \\
SwiD5 & GACCCAAAGTCTCTCTCTCCATC & TGGAGATGTATCACATCCATACG \\
Dp512 & ACTATGCATAACACAGACACACG & GAAGTACGGCAAGGAGCAAC \\
SwiD10 & TTTCGTTCTACCCAGGGAAG & TTTGCTCGTCTGTGATACGC \\
SwiD12 & ATTCTTATTGCCCCAAATAACC & GCCGCTTTTTCTATCTGCATAC \\
SwiD14 & AGACGATCGTTGGTTCATCC & CCGGATAGTTGCTGGAAAAG \\
SwiD15 & TCTTCTTTTTCCCATACAGACTCTC & CTCCCTCTGATTTGGCGTAACT \\
10_14 & CTCTTATAACCAGCACCTCG & CTATTATTCCATCGTCCGTC \\
\hline
\end{tabular}

